\title{
Características da judicialização do acesso a medicamentos no Brasil: uma revisão sistemática
}

I 1 Izamara Damasceno Catanheide, ${ }^{2}$ Erick Soares Lisboa,

${ }^{3}$ Luis Eugenio Portela Fernandes de Souza I

Resumo: O Brasil reconhece o acesso a medicamentos como parte do direito à saúde $\mathrm{e}$ adota políticas públicas para propiciar sua garantia. No entanto, a Política Nacional de Assistência Farmacêutica não tem conseguido atender a essa demanda. Em parte, por isso, tem crescido o recurso ao Poder Judiciário para a obtenção desses medicamentos no país. Com o objetivo de conhecer as características das ações judiciais, realizou-se uma revisão com busca sistemática dos estudos de natureza empírica, publicados entre 1988 e 2014, sobre a judicialização do acesso a medicamentos no Brasil. Os 53 estudos revisados apresentam achados que ajudam a compreender o fenômeno: as liminares são concedidas na quase totalidade dos casos; parcela considerável das ações poderia ter sido evitada caso fossem observadas as alternativas terapêuticas do SUS. Os estudos revisados não permitem afirmar nem negar que os valores gastos com a compra de medicamentos demandados judicialmente comprometam o orçamento do SUS. Enfim, as pesquisas realizadas permitem que se compreenda melhor o fenômeno da judicialização, embora seja recomendável a realização de outros estudos que descrevam características ainda pouco definidas da judicialização do acesso a medicamentos no Brasil.

> Palavras-chave: judicialização; medicamentos; direito à saúde.

\author{
1 Universidade Federal da Bahia, \\ Instituto de Saúde Coletiva. \\ Salvador-BA, Brasil (izamaradc@ \\ gmail.com). \\ 2 Universidade Federal da \\ Bahia, Instituto de Saúde \\ Coletiva. Salvador-BA, Brasil (es. \\ lisboa18@gmail.com). \\ ${ }^{3}$ Universidade Federal da \\ Bahia, Instituto de Saúde \\ Coletiva. Salvador-BA, Brasil \\ (luiseugeniodesouza@gmail. \\ com).
}

Recebido em: 15/10/2015 Aprovado em: 29/06/2016 
No Brasil, o acesso a medicamento é parte do direito à saúde, por se tratar de um bem importante para o cuidado, por seu potencial para prevenir e curar doenças ou até salvar vidas (WANNMACHER, 2010). A garantia do acesso, contudo, é uma questão complexa que depende do contexto econômico, político e social. A situação mundial revela uma grave iniquidade no acesso: apenas $10 \%$ da população mundial consomem $90 \%$ da produção de medicamentos (OLIVEIRA et al., 2007).

De modo geral, a melhoria do acesso está associada à das condições socioeconômicas, especialmente da distribuição de renda, mas também à capacidade de financiamento dos sistemas de saúde, ao uso racional dos medicamentos e à eficiência na gestão dos recursos (MACHADO-DOSSANTOS, 2001). Ademais, características próprias da área farmacêutica representam desafios à garantia do acesso: a complexidade da seleção de quais produtos ofertar, a opacidade dos mecanismos de definição de preços e as dificuldades de desenvolvimento de ações efetivas para promover o uso racional.

A Política de Assistência Farmacêutica do Sistema Único de Saúde (SUS) define as diretrizes de gestão para assegurar o fornecimento e o uso racional de medicamentos. Atualmente, encontra-se organizada em três componentes: básico - medicamentos para o tratamento de doenças mais prevalentes; estratégico - medicamentos para o tratamento de endemias e Aids; e especializado medicamentos cuja utilização depende da observação de Protocolos Clínicos e Diretrizes Terapêuticas (BRASIL, 2011).

Apesar de tecnicamente consistente, a Política de Assistência Farmacêutica não tem conseguido atender às necessidades de acesso a medicamentos, por uma série de fatores, entre os quais se podem citar: o desconhecimento ou a não adesão dos prescritores às listas de medicamentos disponíveis, as falhas na oferta tempestiva e a pressão do setor produtivo em prol do uso de fármacos não contemplados nas listas.

As dificuldades de acesso explicam, em parte, o recurso dos usuários do SUS ao Poder Judiciário. Conforme salienta Farias (2010), a intervenção do Poder Judiciário, mediante determinações à Administração Pública, almeja, primordialmente, a concretização dos direitos previstos na Constituição. Essa intervenção pode ser chamada de judicialização, quando, por sua magnitude ou 
abrangência, implica a transferência para o Judiciário de decisões que caberiam aos Poderes Executivo e Legislativo (MACHADO, 2010).

As ações judiciais que pleiteiam o acesso a medicamentos têm tomado grande vulto, causando impactos significativos na estruturação, no financiamento e na organização do sistema de saúde. Iniciaram-se com ações que exigiam o fornecimento de medicamentos para o tratamento da Aids, estratégia bem-sucedida, que se estendeu, a seguir, para as mais variadas doenças (SANT’ANA et al., 2011).

O tema envolve questôes legais, éticas, políticas, econômicas e sociais, no que se refere ao dever do Estado, à escassez dos recursos e ao conflito entre direitos individuais e direitos coletivos (VIEIRA; ZUCCHI, 2007; PEPE et al., 2010a; MARQUES, 2011; SILVA, 2011). Apesar de ser um fenômeno relativamente recente, a judicialização já motivou a redação de uma grande quantidade de teses, dissertações e artigos. Nesse sentido, parece pertinente e interessante a realização de uma síntese dos estudos, em especial os empíricos, que analisaram a situação fática da judicialização.

Com esse fim, realizou-se uma revisão com busca sistemática de estudos, tendo como questão central: como se caracterizam as demandas judiciais de medicamentos no país, de acordo com os achados do conjunto dos estudos empíricos publicados?

A revisão adotou como referência o Manual de Indicadores de Avaliação e Monitoramento das Demandas Judiciais de Medicamentos (PEPE et al., 2011), que sugere uma série de características das ações judiciais e de seus autores. Assim, para delimitar o problema, a questão específica deste estudo é: como se caracteriza empiricamente a judicialização, levando-se em conta as características sociodemográficas do autor da ação e os aspectos processuais, políticoadministrativos e médico-sanitários das ações judiciais?

Assim, o objetivo deste estudo é caracterizar a judicialização do acesso a medicamentos no Brasil, considerando-se os aspectos processuais, médicosanitários e político-administrativos das ações, assim como algumas características dos autores das ações.

\section{Método}

Para realizar a revisão com busca sistemática dos estudos sobre a judicialização do acesso a medicamentos no Brasil, adotou-se um método estruturado em seis 
etapas (COOPER; HEDGES, 2009): (a) formulação do problema, através de uma pergunta de investigação; (b) pesquisa na literatura - identificação das fontes a serem consultadas, definição de palavras-chave, estratégia de busca, estabelecimento de critérios de inclusão e exclusão; (c) avaliação da qualidade dos estudos; (d) análise dos dados; (e) interpretação dos resultados; e (f) apresentação das conclusōes.

Utilizaram-se como fontes as bases Lilacs, Scielo, Medline, o banco de teses e dissertaçōes da Capes, o Google Acadêmico, além das referências dos estudos selecionados. Foram adotadas as seguintes palavras-chave: medicamentos, assistência farmacêutica, direito, judicialização, ações judiciais, decisōes judicias, demandas judiciais, health, medicine, judicial, legal, e/ou Brazil.

As palavras-chave foram combinadas em estratégias de busca específicas para cada base. Na Lilacs, Scielo e Google Acadêmico, foram aplicadas as seguintes combinações: judicializacao AND medicamento; judici\$ AND medicament\$; judici\$ AND assistência farmacêutica; direito AND assistência farmacêutica. $\mathrm{Na}$ Medline: (("medicine"[MeSH Terms] OR "medicine"[All Fields]) AND judicial[All Fields] AND ("brazil"[MeSH Terms] OR "brazil"[All Fields]) judicial[All Fields] AND ("brazil"[MeSH Terms] OR "brazil"[All Fields]) right[All Fields] AND ("health"[MeSH Terms] OR "health"[All Fields])) AND ("brazil"[MeSH Terms] OR "brazil"[All Fields]) AND legal[All Fields]). E no portal da Capes: medicamentos judicialização; medicamentos açōes judiciais; medicamentos decisões judiciais; medicamentos demandas judiciais; assistência farmacêutica judicial. E nas listas de referências, em cada estudo selecionado foram pesquisadas as palavras judicia ( 1 ; is; lização; lisation), medicamento (s), right to health Brazil.

Foram incluídos trabalhos que: (a) abordavam o acesso a medicamentos no SUS pela via judicial unicamente ou administrativa e judicial; (b) foram publicados entre 1988 (depois da promulgação da Constituição federal) e 2014; (c) estavam disponíveis na íntegra; (d) escritos em português ou inglês. Foram excluídos os estudos sobre a judicialização do acesso a transplantes, cirurgias, exames e internações.

As buscas foram realizadas, inicialmente, em outubro e novembro de 2012 e, mais tarde, atualizadas em setembro de 2015, utilizando-se os mesmos critérios metodológicos. No total, foram localizados 3.339 estudos. Desses, pelo título, 2.924 foram excluídos, ou por não tratarem da judicialização da saúde, ou por 
duplicidade na mesma fonte, ou por não estarem disponíveis na íntegra. Foram ainda removidos 152 estudos repetidos em fontes diferentes.

Os 263 estudos restantes foram analisados com base na leitura do resumo. Foram, então, excluídos aqueles que tratavam da judicialização da saúde, mas não abordavam especificamente o acesso a medicamentos ou não apresentavam o estudo completo. Nessa etapa, foram excluídos 134 estudos. Dos 129 estudos, os que foram publicados como artigos em revistas indexadas foram submetidos a uma análise da qualidade científica. Como proxi da qualidade, foi utilizada a classificação do Qualis Periódicos/Capes. Vinte artigos, publicados em periódicos classificados como C, foram, então, excluídos, tendo sido mantido 109 artigos, publicados em periódicos $\mathrm{A}$ ou $\mathrm{B}$.

Os 109 estudos foram submetidos à verificação de duplicidade entre dissertações e teses, de um lado, e artigos publicados, de outro. Foram identificados 16 artigos originados de 12 dissertações ou teses, que foram, então, excluídas. Em um caso, manteve-se a dissertação (FIGUEREDO, 2010) e foi excluído o artigo, pois o primeiro documento apresentava dados empíricos adicionais que permitiam auxiliar na caracterização das demandas judiciais. Após essa última etapa da exclusão, restaram 96 estudos.

Foram, a seguir, buscados nas referências desses 96 documentos, outros estudos que pudessem interessar à pesquisa. Foram selecionados, então, mais 19 estudos, entre artigos, dissertações e capítulos de livros, chegando-se a um total de 115 documentos. Destes, foram identificados aqueles de natureza empírica, resultando em 77 estudos, dos quais 22 foram excluídos por não possibilitarem a quantificação dos dados de interesse para esta pesquisa. A seguir, foram excluídos dois trabalhos que apresentavam dados já incluídos nesta revisão com base em outros estudos selecionados. Assim, ao final, como mostra a figura 1, foram retidos 53 estudos para a caracterização da judicialização do acesso a medicamentos.

Para a análise, foi aplicado um instrumento elaborado a partir do Manual de Indicadores de Avaliação e Monitoramento das Demandas Judiciais de Medicamentos (PEPE et al., 2011), que relaciona 30 indicadores, categorizados em quatro dimensões: características sociodemográficas do autor da ação e características processuais, político-administrativas e médico-sanitárias das ações judiciais.

Dos 30 indicadores relacionados no Manual, foram selecionados dez, que trazem as informaçōes essenciais para caracterizar o estágio atual da judicialização do acesso a medicamentos no Brasil. 


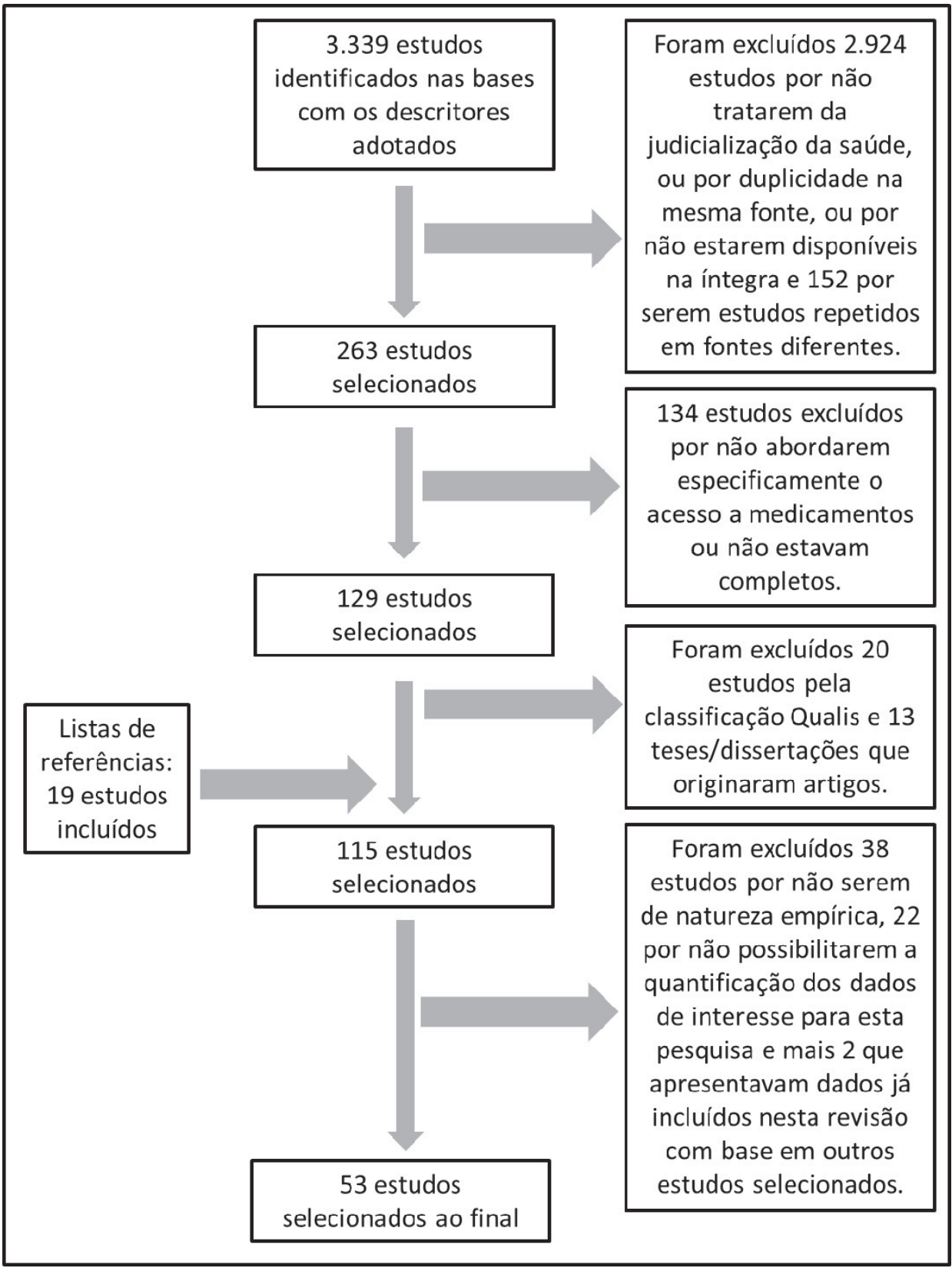

Objetivamente, este estudo contempla as seguintes variáveis: renda mensal familiar e ocupação do autor da ação judicial; proporção de concessão de liminar e exigência judicial de documentos além da prescrição médica; proporção de 
prescrições pelo nome genérico, razão de gasto com medicamentos demandados judicialmente e existência de alternativa terapêutica no Sistema Único de Saúde; registro do medicamento na Agência Nacional de Vigilância Sanitária, proporção de medicamentos por componente da Política de Assistência Farmacêutica e proporção de ações judiciais que demandam ao menos um medicamento que esteja fora dos componentes da Política de Assistência Farmacêutica.

Os dados foram tabulados em planilha Excel ${ }^{\circledR}$ para análise por categoria, de acordo com cada um dos indicadores avaliados.

\section{Resultados e discussão}

\section{Características dos autores}

Dos 53 estudos analisados, nove abordam direta ou indiretamente a renda mensal familiar.

Anjos (2009), ao analisar 42 processos judiciais, identifica que 40 autores (95,2\%) encontravam-se nas classes B (média-alta) e C (média) e dois (4,8\%), na classe A (alta).

Na pesquisa de Pessoa (2007), 93,7\% ( $\mathrm{n}=788)$ dos processos não continham informação sobre renda. Entre os que continham, 0,5\% ( $\mathrm{n}=04)$ dos indivíduos possuíam renda maior que três salários mínimos; $3 \%(\mathrm{n}=25)$, na faixa de dois a três; $2,1 \%(\mathrm{n}=18)$, até um salário e $0,7 \%(\mathrm{n}=06)$ não possuíam renda.

No estudo de Ronsein (2010), há informações sobre a renda para 28,2\% $(\mathrm{n}=201)$ dos indivíduos, dos quais $6,6 \%(\mathrm{n}=47)$ possuíam renda inferior a um salário mínimo; $13,8 \%(\mathrm{n}=98)$, de um a menos que dois e 7,8\% ( $\mathrm{n}=56)$, de dois a mais salários mínimos.

Dos processos analisados por Provin (2011), identifica-se a renda em 90,6\% $(\mathrm{n}=1.249)$ dos casos, apresentando a seguinte distribuição de acordo com o número de salários mínimos: 71,3\% $(\mathrm{n}=89)$, de zero a três; 33,4\% $(\mathrm{n}=417)$, de três a seis; $32,6 \%(n=407)$, de seis a nove e $26,9 \%(n=336)$, acima de nove salários mínimos.

Chieffi e Barata (2009) utilizam o Índice Paulista de Vulnerabilidade Social, indicador que agrega à renda outros dados referentes à escolaridade e ao ciclo de vida familiar, para estratificar as áreas do município de São Paulo. Identificam que $2.136(73 \%)$ autores de demandas judiciais residiam nos estratos em áreas com nenhuma, muito baixa ou baixa vulnerabilidade. 
Vieira e Zucchi (2007) pautam sua análise no índice de exclusão/inclusão social (IEX), composto por vários indicadores, incluindo a renda do chefe de família. Das 133 ações judiciais, 87\% ( $\mathrm{n}=116)$ foram distribuídas conforme o IEX de distintas áreas do município de São Paulo. Revelou-se que 63\% (n=73) dos indivíduos eram domiciliados em área com menor exclusão social.

Menicucci e Machado (2010) utilizam o índice de vulnerabilidade à saúde para evidenciar as desigualdades no perfil epidemiológico de grupos sociais no município de Belo Horizonte. Identificam que 19,2\% ( $\mathrm{n}=48)$ dos autores residiam em áreas com alto e muito alto risco, 42,4\% ( $\mathrm{n}=106)$, em áreas de médio risco e $38,4 \%$ ( $n=96)$, em áreas de baixo risco.

Lima (2012), analisando 174 ações, identifica que 63 autores (66,6\%) apresentam renda mensal de até três salários mínimos e 34 autores $(33,3 \%)$ têm renda inferior ou igual a um salário mínimo.

$\mathrm{Na}$ análise de Marçal (2012), em apenas 41 processos (6,3\%) constava a renda mensal dos autores, dos quais $20(48,8 \%)$ têm renda de meio até um salário mínimo, 15 (36,6\%), de um até três salários mínimos e 6 (14,6\%), de mais de três salários mínimos.

Em suma, os resultados quanto à renda dos autores das ações não permitem uma conclusão inequívoca. De um lado, três estudos (VIEIRA; ZUCCHI, 2007; CHIEFFI; BARATA, 2009, MENICUCCI; MACHADO, 2010) indicam que pessoas de classes sociais altas ou que residem em áreas de baixa vulnerabilidade ou ainda de médio ou baixo risco à saúde foram as que mais recorreram à via judicial para acesso a medicamentos. De outro, um estudo (PROVIN, 2011) que analisa 1.249 processos em Goiânia, em um período de três anos, aponta que mais de $70 \%$ dos autores tinham renda de zero a três salários mínimos.

Além disso, a maioria dos estudos apresenta limites metodológicos: Anjos (2009) analisa o perfil de apenas 42 autores de demandas judiciais; Vieira e Zucchi (2007) restringem-se a 133 autores de ações; Marçal (2012) identifica a renda em apenas 6,3\% dos processos; Chieffi e Barata (2009), apesar de terem analisado um número maior de processos $(\mathrm{n}=2.925)$, limitam seu estudo às ações movidas contra a secretaria estadual de saúde de São Paulo, por moradores da capital; e Menicucci e Machado (2010) analisam apenas 250 autores domiciliados em Belo Horizonte. 
Sendo assim, ainda que possam revelar indícios, especialmente no Estado

de São Paulo, esses achados são insuficientes para afirmar que as demandas judiciais, no Brasil, têm como autores indivíduos com um ou outro nível de renda. Em consequência, não se pode afirmar se a judicialização contribui ou não para aprofundar a iniquidade social no acesso a medicamentos.

No que se refere à ocupação, os aposentados ou pensionistas são apontados como os autores mais frequentes das ações judiciais, em proporções que vão de 8,9\% a 38,1\%, nos quatro estudos (VIEIRA; ZUCCHI, 2007; PESSOA, 2007; RONSEIN, 2010; MACHADO et al., 2011) que trataram da questão. Outro estudo (MENICUCCI; MACHADO, 2010) que também abordou a ocupação dos autores identificou que aposentados, funcionários públicos e profissionais liberais formavam a maioria, com $23 \%$ do total de autores $(n=56)$. São citadas ainda como ocupações: professor, estudante, do lar e desempregado. Contudo, depois de aposentados, o grupo com maior número de autores é o de "outras ocupações”. Com esses dados, não se pode definir o perfil da ocupação dos autores das demandas judiciais.

\section{Características processuais}

Dos 18 estudos que calcularam a proporção de concessão da liminar ou antecipação de tutela, 13 (SANT'ANA et al., 2011; SILVA, 2011; MENICUCCI; MACHADO, 2010; MARÇAL, 2012; CAMARGO, 2011; PEREIRA; PEPE, 2014; MARQUES; DALLARI, 2007; PEPE et al., 2010b; VENTURA et al., 2010; DELDUQUE; MARQUES, 2011; NOGUEIRA, 2011; SARTORI JR. et al., 2012; BLOOD, 2013) encontraram 90\% ou mais de liminares concedidas. Seis estudos (SANT'ANA et al., 2011; SILVA, 2011; PEPE et al., 2010b; VENTURA et al., 2010; SARTORI JR. et al., 2012; BLOOD, 2013) identificaram a antecipação de tutela em 100\% dos casos. Os estudos que encontraram menores proporções foram o de Paula e Saturnino (2010), no qual a concessão de liminar situa-se em torno de 50\% dos casos, Lopes et al. (2014), com 57,9\%, Ramos e Ferreira (2013), com 78,3\% e Coelho et al. (2014), com $82,5 \%$ de liminares concedidas.

Apenas cinco estudos estimaram a proporção de ações judiciais contendo documentos adicionais, além da prescrição de medicamento. Em três casos, o documento adicional foi o relatório médico, inserido nos autos em 99,4\% ( $\mathrm{n}=177)$ 
1344 dos processos analisados por Camargo (2011), em 81,2\% ( $\mathrm{n}=13)$ dos analisados por Silva (2011) e em 100\% ( $\mathrm{n}=27)$ dos examinados por Sant'ana et al. (2011). Lima (2012) encontrou laudos médicos de medicamentos excepcionais ou de exames complementares em 97,7\% $(n=170)$ das açôes. Pereira e Pepe (2014) notaram documentos adicionais em 100\% $(\mathrm{n}=450)$ das ações, o que atribuíram a uma peculiaridade da Justiça do Paraná.

Vê-se, assim, que, na prática judicial, a concessão de liminar é regra, e a prova necessária e suficiente de que o autor precisa do medicamento requerido é a prescrição de um médico.

Para Romero (2010, p. 40), os juízes justificam a proeminência da prescrição médica, por acreditarem que "é o médico que entende das necessidades do paciente". Todavia, como alertam Bonfim (2008) e Marques (2011), deve-se questionar esse entendimento dos juízes, pois se sabe que ocorrem falhas na prescrição médica. A busca de uma segunda opinião ou de um parecer de uma junta médica, muitas vezes, é a conduta mais prudente. Infelizmente, como demonstram Gomes e Amador (2015), os magistrados têm dado, de modo geral, ganho de causa aos cidadãos que solicitam o acesso aos medicamentos por via judicial com base exclusiva na prescrição de um médico.

Nessa linha, Sant'ana et al. (2011), analisando as receitas médicas, viram que nenhuma cumpriu os preceitos de boas práticas de prescrição, definidos em normas legais (BRASIL, 1973; 1998; 1999; CFF, 2001). Todavia, todas foram deferidas pelo Poder Judiciário. Prescrições ilegíveis, sem assinatura do profissional, sem data, sem o nome genérico (no âmbito do SUS), etc., foram suficientes para embasar a sentença judicial.

Se o Judiciário não exige outros documentos probatórios da necessidade de uso do medicamento, deveria, ao menos, observar se a prescrição médica cumpre os requisitos legais, já que tem exercido o papel de árbitro do acesso aos medicamentos (LEITÃO et al., 2014). Os prescritores, por sua vez, precisam se conscientizar da necessidade de cumprir os preceitos legais para evitar problemas para os usuários e para si mesmos.

\section{Características médico-sanitárias}

Em 48 estudos, do total de medicamentos prescritos, a proporção dos indicados pelo nome genérico situou-se na faixa de 19,5\% a 48,8\%. Em apenas quatros 
estudos (LIMA, 2012; MARÇAL, 2012; ROMERO, 2008; CONTI, 2013)

mais da metade dos medicamentos foi prescrita pelo nome genérico. Registrese que a Lei Federal n ${ }^{\circ}$ 9.787, de 1999, recomenda que todas as prescrições no âmbito do SUS sejam feitas pelo nome genérico, o que, pelo observado, não está sendo cumprido.

Leite et al. (2009) chamam a atenção que as prescrições pelos nomes comerciais representam a concessão de um privilégio a determinadas empresas e contribuem para aumentar os custos da assistência farmacêutica. Observaram uma variação de $\mathrm{R}$ \$24,00 a $\mathrm{R}$ \$ 250,00 para um mesmo medicamento nas ações que analisaram. Ademais, a prescrição pelo nome genérico é uma estratégia para o uso racional que proporciona maior segurança para o usuário. Além disso, tem a vantagem de estimular a compra de medicamentos mais baratos e promover a melhor utilização dos recursos públicos.

A razão de gastos entre medicamentos demandados judicialmente e medicamentos de aquisição programada foi calculada em quatro estudos (MENICUCCI; MACHADO, 2010; PEREIRA; PEPE, 2014; CHIEFFI; BARATA, 2010, PEREIRA et al., 2010).

Chieffi e Barata (2010) identificam um gasto de 65 milhões de reais com medicamentos demandados judicialmente da Secretaria da Saúde do Estado de São Paulo no ano de 2006. Como o gasto total com medicamentos foi de 1,2 bilhão de reais, a razão de gastos foi cerca de 0,05 real, ou seja, os gastos impostos judicialmente representaram, aproximadamente, $5 \%$ dos gastos programados.

Menicucci e Machado (2010) encontraram a mesma razão (0,05 real), e a mesma proporção, ao identificarem gastos de 1,7 milhão de reais pela via demanda judicial e de 32 milhões de reais na aquisição programada em Belo Horizonte.

Razões mais elevadas, significando um maior comprometimento de recursos financeiros com as demandas judiciais, foram encontradas em Santa Catarina por Pereira et al. (2010). Os autores identificaram que foram gastos, nos anos de 2003 e 2004, o total de $\mathrm{R} \$ 93,5$ milhôes na compra de medicamentos pela via judicial em um total de $\mathrm{R} \$ 987$ milhões gastos com medicamentos, resultando na razão de 0,1 real aproximadamente, entre medicamentos demandados judicialmente e aqueles de aquisição programada, Em outras palavras, os gastos impostos judicialmente representaram $10 \%$ dos gastos programados. 
Considerando apenas os recursos do tesouro estadual do Paraná, Pereira e Pepe (2014) encontraram uma razão de gastos de 1,78 real. Ou seja, a secretaria da saúde gastou com demandas judiciais ( $\mathrm{R}$ \$ 35 milhões), quase o dobro do que tinha programado para adquirir com recursos próprios ( $\mathrm{R}$ \$ 19,6 milhōes).

Além da razão de gasto, Chieffi e Barata (2010) compararam o gasto por paciente atendido pela via judicial com o gasto por paciente cadastrado no Programa de Medicamentos Excepcionais, mostrando que o primeiro somou de 18 mil reais, enquanto o segundo totalizou 2,2 mil reais.

Outros estudos (VIEIRA e ZUCCHI, 2007; PROVIN, 2011; PAULA e SATURNINO, 2010; CAMARGO, 2011; LEITE et al., 2009; CUNHA, 2011; LOPES et al., 2010) apontam os montantes gastos com a compra de medicamentos demandados por via judicial, porém não informam a razão de gastos, nem proporção do gasto total, nem os gastos por paciente. Entre esses, Lopes et al. (2010) mostram a inadequação do gasto, ao identificar que mais de seis milhōes de reais foram aplicados, pelo Estado de São Paulo, na compra de antineoplásicos, demandados pela Justiça, que não tinham evidência científica de eficácia para a indicação terapêutica realizada.

Ainda que esses resultados não informem se as demandas judiciais estão comprometendo o orçamento do SUS, mostram que os valores são altos e que nem sempre destinam-se a garantir o acesso a medicamentos de segurança e eficácia comprovadas.

A proporção de medicamentos demandados que têm alternativa terapêutica no Sistema Único de Saúde foi estimada em quatro estudos. Provin (2011) revela que havia alternativa disponível para 41,7\% $(\mathrm{n}=741)$ das drogas demandadas judicialmente, enquanto Machado et al. (2011) observam a existência de alternativa para $80 \%(\mathrm{n}=806)$ dos medicamentos. Nos outros dois estudos (VIEIRA; ZUCCHI, 2007; VIEIRA; ZUCCHI, 2009), identificou-se que $73 \%(\mathrm{n}=43)$ e $96 \%(\mathrm{n}=26)$ dos medicamentos demandados tinham alternativas no SUS para as doenças referidas nos processos.

Em que pese o número reduzido de estudos, a quantidade de medicamentos avaliados permite afirmar que a disponibilidade de alternativas terapêuticas mostra que a gestão do SUS não é completamente omissa. Ações judicias poderiam ser evitadas se os prescritores observassem as listas de medicamentos do SUS ou se os juízes consultassem os gestores da saúde, antes de tomarem uma decisão (VIEIRA; ZUCCHI, 2007). 
Os 14 estudos que observaram a proporção de medicamentos registrados na Agência Nacional de Vigilância Sanitária (Anvisa) identificaram que $90,4 \%$ dos medicamentos demandados judicialmente possuíam registro na agência reguladora. Em 13 estudos, o número de medicamentos sem registro identificados nos processos judiciais foi de zero a 14. Um estudo (MACHADO et al., 2011) encontrou 85 fármacos sem registro, apenas 4,8\% do total. Em suma, são poucos os casos de demandas judiciais de medicamentos não registrados oficialmente no Brasil.

Vale mencionar que os estudos sobre a judicialização têm ressaltado a necessidade da observância pelos prescritores e pelos juízes do registro oficial do medicamento, procedimento que visa proteger a saúde da população (SANT'ANA et al., 2011; FIGUEREDO, 2010; RONSEIN, 2010; ROMERO, 2010; ROMERO, 2008; FAGUNDES; CHIAPPA, 2010; PEREIRA, 2007; MARQUES; DALLARI, 2007). Pepe et al. (2010a) sugerem que a prescrição de um medicamento sem registro, fornecido por via judicial, pode ser uma estratégia dos produtores para pressionar a autoridade reguladora.

A proporção de medicamentos por componente da Política de Assistência Farmacêutica é identificada em 20 estudos (SANT'ANA et al., 2011; FIGUEREDO, 2010; PESSOA, 2007; RONSEIN, 2010; PROVIN, 2011; CHIEFFI; BARATA, 2009; MENICUCCI; MACHADO, 2010; MARÇAL, 2010; MACHADO et al., 2011; PEREIRA; PEPE, 2014; RAMOS; FERREIRA, 2013; CONTI, 2013; LEITE et al., 2009; PEREIRA et al., 2010; MACEDO et al., 2011; MESSEDER et al., 2005, SILVA; CORTE, 2011; PAULA, 2010; MACHADO et al., 2010; FERREIRA, 2007). Nesse conjunto, foram classificados 8.659 medicamentos, dos quais $63 \%$ (5.461) pertencem ao componente especializado, 34\% (2.906) ao componente básico e apenas 3\% (292) ao componente estratégico. Note-se que, se a maior proporção de medicamentos demandados judicialmente pertence ao componente especializado, não deixa de ser expressiva a parte do componente básico. Já o estudo de Coelho et al. (2014) aponta que 54,5\% dos medicamentos judicializados pertenciam à Rename 2013.

A demanda judicial de medicamentos pertencentes ao rol da assistência farmacêutica evidencia a existência de problemas gerenciais: falta de medicamentos, barreiras ao acesso, demora na avaliação das solicitações administrativas ou 
1348 desatualização dos Protocolos Clínicos e Diretrizes Terapêuticas (SANT'ANA et al., 2011; VIEIRA; ZUCCHI, 2007; RONSEIN, 2010; CHIEFFI; BARATA, 2009; MARQUES; DALLARI, 2007; ROMERO, 2008; MACEDO et al., 2011; MESSEDER et al., 2005).

No caso do componente especializado, essa demanda pode ser decorrente da recusa, pela gestão do SUS, da dispensação de medicamentos com base em prescrições que não obedecem aos Protocolos Clínicos e Diretrizes Terapêuticas (RONSEIN, 2010). Pode ser ainda que medicamentos pertencentes às listas do SUS se tornem objetos de demanda judicial não por si mesmos, mas pelo fato de constarem de ações que requerem um medicamento não incluído nas listas.

Uma hipótese para explicar esses resultados é o desconhecimento ou a não adesão dos prescritores às listas do SUS (PEREIRA et al., 2010; SILVA e CORTE, 2011). Outra hipótese é que a solicitação judicial de medicamentos fora das listas do SUS seja estratégia dos produtores, associados a médicos ou a associações de usuários, para introduzir medicamentos nos protocolos do SUS (CHIEFFI; BARATA, 2010; LEITE; MAFRA, 2010).

Com efeito, dois estudos (PEPE et al., 2010b; SANT'ANA, 2009), ao analisar a proporção de açôes judiciais que demandam ao menos um medicamento que esteja fora dos componentes da assistência farmacêutica, encontram, respectivamente, taxas de $80,6 \%$ e $81,5 \%$. É possível que esses medicamentos não pertencentes às listas oficiais tenham sido os motivadores das demandas judiciais, as quais resultaram em liminares ou sentenças determinando o fornecimento de todos os medicamentos constantes da prescrição médica.

Outros 14 estudos, em vez de identificar o percentual de ações, apontam a proporção de medicamentos que não pertenciam a nenhuma lista oficial. Em quatro (VIEIRA; ZUCCHI, 2007; DELDUQUE; MARQUES, 2011; BORGES; UGÁ, 2010; PAULA, 2010), os percentuais ficaram entre $37,7 \%$ e $48 \%$, em um total de 418 medicamentos. Nos outros dez (FIGUEREDO, 2010; RONSEIN, 2010; PROVIN, 2011; CHIEFFI; BARATA, 2009; MENICUCCI; MACHADO, 2010; MACHADO et al., 2011; LEITE et al., 2009; PEREIRA et al., 2010; MACEDO et al., 2011; SILVA; CORTE, 2011), a proporção variou de $55 \%$ a $77,5 \%$, perfazendo um total de 21.567 medicamentos que não integravam as listas do SUS. 
Enfim, os medicamentos pertencentes aos componentes da assistência farmacêutica do SUS estão presentes de modo significativo nas ações judiciais, mas há um predomínio de medicamentos que não fazem parte das listas oficiais.

\section{Considerações finais}

Os 53 estudos empíricos revisados revelam tanto o que se sabe sobre o fenômeno atual da judicialização do acesso a medicamentos no Brasil, quanto os aspectos pouco esclarecidos e as lacunas do conhecimento.

Ao menos, quatro pontos estão bem estabelecidos. Primeiro: a prescrição médica é a prova necessária e suficiente para embasar a sentença judicial no deferimento de liminares. Segundo: predomina a prescrição pelo nome comercial, em detrimento da denominação genérica. Terceiro: a maioria das ações teria sido evitada, caso fossem observadas as alternativas terapêuticas presentes nas listas do SUS. Quarto: os medicamentos sem registro na agência reguladora são exceção como objetos de ações judiciais.

Os pontos pouco esclarecidos são diversos. Embora vários estudos indiquem que a judicialização favorece indivíduos com boas condições socioeconômicas, há resultados contraditórios, que estão a exigir estudos mais abrangentes. De modo similar, não se pode afirmar nem negar que os valores gastos com a compra de medicamentos demandados judicialmente comprometam o orçamento do SUS. Tampouco se sabe, ao certo, se os medicamentos do elenco do SUS foram incluídos nas ações judiciais em decorrência de falhas na gestão ou apenas por constarem da mesma prescrição de um medicamento não pertencente que motivou a demanda. De modo similar, não está claro se a demanda judicial por medicamentos fora dos componentes da assistência farmacêutica resulta da não adesão dos prescritores às listas oficiais, de estratégias da indústria e/ou da desatualização das listas.

Por fim, como lacunas no conhecimento sobre a situação concreta da judicialização do acesso a medicamentos no Brasil, esta revisão identifica: os desfechos da utilização do medicamento demandado judicialmente, o grau de adesão dos prescritores às listas oficiais e a participação da indústria e das associações de portadores de doenças nesse processo.

Sendo assim, para um diagnóstico mais acurado da realidade da judicialização do acesso a medicamentos no Brasil, é recomendável a realização tanto de estudos 
abrangentes, envolvendo as três esferas de gestão do SUS, em todas as regiōes do país, conforme já indicado por Pepe et al. (2011), quanto de pesquisas específicas, focadas nos possíveis determinantes do fenômeno, incluindo as motivações dos demandantes. Certamente, tais estudos serão fontes de sugestões de medidas de enfrentamento da situação e de garantia do direito ao acesso a medicamentos. ${ }^{1}$

\section{Referências}

ANJOS, R. S. Judicialização e equidade no tratamento da Hepatite C: estudo de caso sobre o tratamento com Interferona Alfa em um serviço de referência do SUS em Porto Alegre, RS. 2009. 83 p. Dissertação (Mestrado em Gestão Tecnológica em Saúde) - Faculdade de Medicina, Universidade Federal do Rio Grande do Sul, Porto Alegre.

BLOOD, R. L. P. Y. Consideraçôes sobre a judicialização da assistência farmacêutica na comarca de Ponta Grossa-PR no ano de 2011. 2013. 128 p. Dissertação (Mestrado em Ciências Sociais Aplicadas) - Universidade Estadual de Ponta Grossa, Ponta Grossa.

BONFIM, R. L. D. Agenda Única de Saúde: a busca do acesso universal e a garantia do direito à saúde. 2008. 164 p. Tese (Doutorado em Saúde Coletiva) - Instituto de Medicina Social, Universidade do Estado do Rio de Janeiro, Rio de Janeiro.

BORGES, D. C. L.; UGÁ, M. A. D. Conflitos e impasses da judicialização na obtenção de medicamentos: as decisões de $1^{\text {a }}$ instância nas ações individuais contra o estado do Rio de Janeiro. Cad. Saúde Pública. Rio de Janeiro, v. 26, n. 1, p. 59-69, 2010.

BRASIL. Assistência farmacêutica no SUS. Brasília: Conselho Nacional de Secretários de Saúde, 2011. 186p.

. Lei no 5.991, de 17 de dezembro de 1973. Dispóe sobre o controle sanitário do comércio de drogas, medicamentos, insumos farmacêuticos e correlatos, e dá outras providências. Diário Oficial [da] República Federativa do Brasil. Brasília, DF, 21 dez. 1973. Portaria no 3.916, de 30 de outubro de 1998. Dispóe sobre a Política Nacional de Medicamentos. Diário Oficial [da] República Federativa do Brasil. Brasília, DF, 10 nov. 1998.

Portaria no 344, de 12 de maio de 1998. Aprova o Regulamento técnico sobre substâncias e medicamentos sujeitos a controle especial. Diário Oficial [da] República Federativa do Brasil. Brasília, DF, 01 fev. 1999.

CAMARGO, I. A. Análise das demandas judiciais para o tratamento da artrite reumatoide no estado de São Paulo. 2011. 139 p. Dissertação (Mestrado em Ciências Farmacêuticas) Universidade de Sorocaba, Sorocaba.

CHIEFFI, A. L.; BARATA, R. B. Açôes judiciais: estratégia da indústria farmacêutica para introdução de novos medicamentos. Rev. Saúde Pública. São Paulo, v. 44, n. 3, p. 421-429, 2010. 
CHIEFFI, A. L.; BARATA, R. B. Judicialização da política pública de assistência farmacêutica e equidade. Cad. Saúde Pública. Rio de Janeiro, v. 25, n. 8, p. 1839-1849, 2009.

COELHO, T. L. et al. Variáveis jurídicas e de saúde no deferimento de liminares por medicamentos em Minas Gerais. Rev. Saúde Pública. São Paulo, v. 48, n. 5, p. 808-816, 2014. CONSELHO FEDERAL DE FARMÁCIA (CFF). A Organização Jurídica da Profissão Farmacêutica. Brasília: CFF, 2001.

CONTI, M. A. Avaliação das demandas judiciais por acesso a medicamentos no Distrito Federal. 2013. 101 p. Dissertação (Mestrado em Ciências da Saúde) - Faculdade de Ciências da Saúde, Universidade de Brasília, Brasília.

COOPER, H. M.; HEDGES, L. V. The Handbook of research synthesis and meta-analysis. New York: The Russel Sage Foundation, 2009.

CUNHA, A. E. A judicialização do direito a saúde no estado de Santa Catarina. 2011. 90 p. Dissertação (Mestrado em Gestão de Políticas Públicas) - Centro de Ciências Sociais e Jurídicas, Universidade do Vale do Itajaí, Itajaí.

DELDUQUE, M. C.; MARQUES, S. B. A judicialização da política de assistência farmacêutica no Distrito Federal: diálogos entre política e o direito. Rev. Eletrol. Tempus Actas Saúde Coletiva. Brasília, v. 5, n. 4, p. 97-106, 2011.

FAGUNDES, P. O.; CHIAPPA, R. Avaliação do uso de medicamentos não registrados no Brasil por meio de ações judiciais no estado de Minas Gerais. In: AITH, F. et al. (Orgs.). Direito Sanitário: saúde e direito, um diálogo possível. Belo Horizonte: ESP-MG, 2010, p. 353-372.

FARIAS, L. C. Mínimo existencial: um parâmetro para o controle judicial para as políticas em saúde. 2010. 149 p. Dissertação (Mestrado em Políticas Sociais e Cidadania) Universidade Católica do Salvador, Salvador.

FERREIRA, M. G. M. Perfil das demandas judiciais para acesso a medicamentos no municipio de Itaperuna: uma contribuição sobre novas perspectivas de acesso racional e igualitário. 2007. 69 p. Dissertação (Mestrado em Saúde da Família) - Universidade Estácio de Sá, Rio de Janeiro.

FIGUEIREDO, T. A. Análise dos medicamentos fornecidos por mandado judicial na Comarca do Rio de Janeiro: a aplicação de evidências científicas no processo de tomada de decisão. 2010. 146 p. Dissertação (Mestrado em Saúde Pública) - Fundação Oswaldo Cruz, Escola Nacional de Saúde Pública Sergio Arouca, Rio de Janeiro.

GOMES, V. S.; AMADOR, T. A. Estudos publicados em periódicos indexados sobre decisões judiciais para acesso a medicamentos no Brasil: uma revisão sistemática. Cad. Saúde Pública. Rio de Janeiro, v. 31, n. 3, p. 451-462, 2015.

LEITÃO, L. C. A. et al. Judicialização da saúde na garantia do acesso ao medicamento. Rev. Salud Pública. Bogotá, v. 16, n. 3, p. 360-370, 2014. 
LEITE, S. N.; MAFRA, A. C. Que direito? Trajetórias e percepções dos usuários no processo de acesso a medicamentos por mandados judiciais em Santa Catarina. Cien. Saúde Coletiva. Rio de Janeiro, v. 15, supl. 1, p. 1665-1672. 2010.

LEITE, S. N. et al. Açôes judiciais e demandas administrativas na garantia do direito de acesso a medicamentos em Florianópolis-SC. Rev. Direito Sanitário. São Paulo, v. 10, n. 2, p. 13-28, 2009.

LIMA, G. S. Demanda judicialde medicamentos e uso de indicadores de avaliação e monitoramento no Estado do Rio de Janeiro. 2012. 132 p. Dissertação (Mestrado em Saúde Pública) - Fundação Oswaldo Cruz, Escola Nacional de Saúde Pública Sergio Arouca, Rio de Janeiro.

LOPES, L. C. et al. Uso racional de medicamentos antineoplásicos e açôes judiciais no estado de São Paulo. Rev. Saúde Pública. São Paulo, v. 44, n. 4, p. 620-628, 2010.

LOPES, L. C. et al. Medicamentos biológicos para o tratamento de psoríase em sistema público de saúde. Rev. Saúde Pública. São Paulo, v. 48, n. 4, p. 651-661, 2014.

MACEDO, E. I.; LOPES, L. C.; BARBERATO-FILHO, S. Análise técnica para a tomada de decisão do fornecimento de medicamentos pela via judicial. Rev. Saúde Pública. São Paulo, v. 45, n. 4, p. 706-713, 2011.

MACHADO, F. R. S. A judicialização da saúde no Brasil: cidadanias e assimetrias. 2010. 187 p. Tese (Doutorado em Saúde Coletiva) - Instituto de Medicina Social, Universidade do Estado do Rio de Janeiro, Rio de Janeiro.

MACHADO, L. R.; RESENDE.; A. L. R.; SATURNINO, L. T. M. Medicamentos especializados na judicialização da saúde: uma análise das demandas judiciais no estado de Minas Gerais. In: AITH, F. et al. (Org.). Direito Sanitário: saúde e direito, um diálogo possível. Belo Horizonte: ESP-MG, 2010, p. 323-338.

MACHADO, M. A. A. et al. Judicialização do acesso a medicamentos no estado de Minas Gerais, Brasil. Rev. Saúde Pública. São Paulo, v. 45, n. 3, p. 590-598, 2011.

MACHADO-DOS-SANTOS, S. C. Melhoria da equidade no acesso aos medicamentos no Brasil: os desafios impostos pela dinâmica da competição "extrapreço". 2001. 180 p. Dissertação (Mestrado em Saúde Pública) - Fundação Oswaldo Cruz, Escola Nacional de Saúde Pública Sérgio Arouca, Rio de Janeiro.

MARÇAL, K. K. S. A judicialização da assistência farmacêutica: o caso Pernambuco 2009 e 2010. 2012. 132 p. Dissertação (Mestrado em Saúde Pública) - Fundação Oswaldo Cruz, Centro de Pesquisas Aggeu Magalhães, Recife.

MARQUES, S. B.; DALLARI, S. G. Garantia do direito social à assistência farmacêutica no estado de São Paulo. Rev. Saúde Pública. São Paulo, v. 41, n. 1, p. 101-107, 2007.

MARQUES, S. B. O direito ao acesso universal a medicamentos no Brasil: diálogos entre o direito, a política e a técnica médica. 2011. 396 p. Tese (Doutorado em Saúde Pública) Faculdade de Saúde Pública, Universidade de São Paulo, São Paulo. 
MENICUCCI, T. M. G.; MACHADO, J. A. Judicialization of health policy in the definition of access to public goods: Individual rights versus collective rights. Brazilian Political Science Review. São Paulo, v. 4, n. 1, p. 33-68, 2010.

MESSEDER, A. M.; OSORIO-DE-CASTRO, C. G. S.; LUIZA, V. L. Mandados judiciais como ferramenta para garantia do acesso a medicamentos no setor público: a experiência do estado do Rio de Janeiro, Brasil. Cad. Saúde Pública. Rio de Janeiro, v. 21, n. 2, p. 525-534, 2005.

NOGUEIRA, D. C. B. A efetivação do direito à saúde: fornecimento judicial de medicamentos. 2011. 165 p. Dissertação (Mestrado em Políticas Públicas) - Universidade Federal do Maranhão, São Luiz.

OLIVEIRA, M. A.; BERMUdEZ, J. A. Z.; OSORIO-DE-CASTRO, C. G. S. Assistência Farmacêutica e acesso a medicamentos. Rio de Janeiro: Fiocruz; 2007. 110p.

PAULA, C. S. Uso offlabel de medicamentos: análise das demandas judiciais no Cemepar e conduta dos farmacêuticos no Paraná. 2010. 145 p. Dissertação (Mestrado em Ciências Farmacêuticas) - Universidade Federal do Paraná, Curitiba.

PAULA, O. S.; SATURNINO, L. T. M. Descrição das demandas do medicamento Etanercepte no estado de Minas Gerais no período de janeiro a abril de 2008. In: AITH, F. et al. (Org.). Direito Sanitário: saúde e direito, um diálogo possível. Belo Horizonte: ESPMG, 2010, p. 339-352.

PEPE, V. L. E. et al. A judicialização da saúde e os novos desafios da gestão da assistência farmacêutica. Cien. Saúde Coletiva. Rio de Janeiro, v. 15, n. 5, p. 2405-2414, 2010a.

PEPE, V. L. E.; VENTURA, M.; OSORIO-DE-CASTRO, C. G. S. (Orgs.). Manual de indicadores de avaliação e monitoramento das demandas judiciais de medicamentos. Rio de Janeiro: Fiocruz, Escola Nacional de Saúde Pública Sergio Arouca, 2011. 65p.

PEPE, V. L. E. et al. Caracterização de demandas judiciais de fornecimento de medicamentos "essenciais" no Estado do Rio de Janeiro, Brasil. Cad. Saúde Pública. Rio de Janeiro, v. 26, n. 3, p. 461-471, 2010b.

PEREIRA, J. G.; PEPE, V. L. E. Acesso a medicamentos por via judicial no Paraná: aplicação de um modelo metodológico para análise e monitoramento das demandas judiciais. Rev. Direito Sanitário. São Paulo, v. 15, n. 2, p. 30-45, 2014.

PEREIRA, J. R. et al. Análise das demandas judiciais para o fornecimento de medicamentos pela Secretaria de Estado da Saúde de Santa Catarina nos anos de 2003 e 2004. Cien. Saúde Coletiva. Rio de Janeiro, v. 15, supl. 3, p. 3551-3560, 2010.

PEREIRA, S. M. P. Da reorientação do modelo assistencial à pressão social: acesso a medicamentos via Sistema Único de Saúde por compra direta no município de Florianópolis - SC. 2007. 112 p. Dissertação (Mestrado em Saúde e Gestão do Trabalho) - Centro de Educação de Ciências da Saúde, Universidade do Vale do Itajaí, Itajaí. 
PESSOA, N. T. Perfil das solicitaçôes administrativas e judiciais de medicamentos impetradas contra a secretaria de saúde do estado do Ceará. 2007. 168 p. Dissertação (Mestrado em Ciências Farmacêuticas) - Departamento de Farmácia, Universidade Federal do Ceará, Fortaleza.

PROVIN, M. P. Demandas judiciais por medicamentos deferidas em Goiânia-GO, Brasil, e seus aspectos financeiros. 2011. Tese (Doutorado em Ciências da Saúde) - Universidade Federal de Goiás, Goiânia.

RAMOS, K. A.; FERREIRA, A. S. D. Análise da demanda de medicamentos para uso offlabel por meio de ações judiciais na secretaria de estado de saúde de Minas Gerais. Rev. Direito Sanitário. São Paulo, v. 14, n. 1, p. 98-121, 2013.

ROMERO, L. C. Judicialização das políticas de assistência farmacêutica: o caso do Distrito Federal. In: Brasil. Textos para discussão. Brasília: Consultoria Legislativa do Senado Federal, 2008, p. 4-48.

ROMERO, L. C. P. A jurisprudência do Tribunal de Justiça do Distrito Federal em ações de medicamentos. Rev. Direito Sanitário. São Paulo, v. 11, n. 2, p. 11-59, 2010.

RONSEIN, J. G. Análise do perfil das solicitaçôes de medicamentos por demanda judicial no Estado de Santa Catarina no periodo de 2005 a 2008. 2010. 215 p. Dissertação (Mestrado em Farmácia) - Centro de Ciências da Saúde, Universidade Federal de Santa Catarina, Florianópolis.

SANT'ANA, J. M. B. et al. Racionalidade terapêutica: elementos médico-sanitários nas demandas judiciais de medicamentos. Rev. Saúde Pública. São Paulo, v. 45, n.4, p. 714721, 2011.

SANT'ANA, R. N. A saúde aos cuidados do judiciário: a judicialização das políticas públicas de assistência farmacêutica no Distrito Federal a partir da jurisprudência do TJDFT. 2009. 159 p. Dissertação (Mestrado em Direito) - Faculdade de Direito, Universidade de Brasília, Brasília.

SARTORI JR., D. et al. Judicialização do acesso ao tratamento de doenças genéticas raras: a doença de Fabry no Rio Grande do Sul. Cien. Saúde Coletiva. Rio de Janeiro, v. 17, n. 10, p. 2717-2728, 2012.

SILVA, A. R. H.; CORTE, E. D. Judicialização na assistência farmacêutica do Sistema Único de Saúde. Ciência em Movimento. Porto Alegre, v. 13, n. 27, p. 19-25, 2011.

SILVA, R. T. B. Conflitos bioéticos decorrentes do acesso a medicamentos órfãos no Brasil: o exemplo da laronidase para o tratamento da mucopolissacaridose tipo I. 2011. 132 p. Tese (Doutorado em Saúde Pública) - Fundação Oswaldo Cruz, Escola Nacional de Saúde Pública Sérgio Arouca, Rio de Janeiro.

VENTURA, M. et al. Judicialização da saúde, acesso à justiça e a efetividade do direito à saúde. Physis: Revista de Saúde Coletiva. Rio de Janeiro, v. 20, n. 1, p. 77-100, 2010. 
VIEIRA, F. S.; ZUCCHI, P. Demandas judiciais e assistência terapêutica no Sistema Único de Saúde. Rev. Assoc. Med. Bras. São Paulo, v. 55, n. 6, p. 672-683, 2009.

VIEIRA, F. S.; ZUCCHI, P. Distorções causadas pelas ações judiciais à política de medicamentos no Brasil. Rev. Saúde Pública. São Paulo, v. 41, n. 2, p. 214-222, 2007.

WANNMACHER, L. Importância dos Medicamentos Essenciais em Prescrição e Gestão Racionais. In: BRASIL. Uso Racional de Medicamentos: temas selecionados. Brasília: Ministério da Saúde, 2010, p. 15-20.

\section{Nota}

${ }^{1}$ I. D. Catanheide concebeu e delineou o artigo, coletou, analisou e interpretou os dados, redigiu o artigo e aprovou sua versão final. E. S. Lisboa coletou, analisou e interpretou os dados, redigiu o artigo e aprovou sua versão final. L. E. P. F. de Souza delineou, revisou criticamente e aprovou a versão do artigo a ser publicada. 
Characteristics of the judicialization of access to medicines in Brazil: a systematic review Brazil recognizes access to medicines as part of the right to health and adopts public policies to provide its guarantee. However, the National Policy on Pharmaceutical Assistance has not been able to meet this demand. In part because of this, there has been a growing recourse to the Judiciary to obtain these medicines in the country. In order to know the characteristics of the lawsuits, a systematic review of the empirical studies published between 1988 and 2014 on the judicialization of access to medicines in Brazil was carried out. The 53 studies reviewed present findings that help to understand the phenomenon: injunctions are granted in almost all cases; A considerable portion of the actions could have been avoided if the therapeutic alternatives of SUS were observed. The studies reviewed do not allow to affirm or deny that the amounts spent on the purchase of drugs demanded judicially compromise SUS budget. Finally, the research carried out allows us to better understand the phenomenon of judicialization, although it is advisable to carry out other studies that describe characteristics that have not yet been defined in the judicialization of access to medicines in Brazil.

> Keywords: judicialization; medicines; right to health. 